\title{
BMJ Open Does case management for patients with heart failure based in the community reduce unplanned hospital admissions? A systematic review and meta-analysis
}

\author{
A L Huntley, R Johnson, A King, R W Morris, S Purdy
}

To cite: Huntley AL, Johnson R, King A, et al. Does case management for patients with heart failure based in the community reduce unplanned hospital admissions? A systematic review and meta-analysis. BMJ Open 2016;6:e010933. doi:10.1136/bmjopen-2015010933

- Prepublication history and additional material is available. To view please visit the journal (http://dx.doi.org/ 10.1136/bmjopen-2015010933).

Received 22 December 2015 Revised 24 March 2016 Accepted 15 April 2016

CrossMark

Centre of Academic Primary Care, School of Social and Community Medicine, University of Bristol, Bristol, UK

Correspondence to Dr AL Huntley; alyson.huntley@bristol.ac.uk

\section{ABSTRACT}

Objectives: The aim of this systematic review of randomised controlled trials (RCTs) and controlled trials (non-RCTs, NRCTs) is to investigate the effectiveness and related costs of case management (CM) for patients with heart failure (HF) predominantly based in the community in reducing unplanned readmissions and length of stay (LOS).

Setting: CM initiated either while as an inpatient, or on discharge from acute care hospitals, or in the community and then continuing on in the community.

Participants: Adults with a diagnosis of $\mathrm{HF}$ and resident in Organisation for Economic Co-operation and Development countries.

Intervention: CM based on nurse coordinated multicomponent care which is applicable to the primary care-based health systems.

Primary and secondary outcomes: Primary outcomes of interest were unplanned (re)admissions, LOS and any related cost data. Secondary outcomes were primary healthcare resources.

Results: 22 studies were included: 17 RCTs and 5 NRCTs. 17 studies described hospital-initiated CM $(\mathrm{n}=4794)$ and 5 described community-initiated CM of HF ( $n=3832$ ). Hospital-initiated CM reduced readmissions (rate ratio $0.74(95 \% \mathrm{Cl} 0.60$ to 0.92$)$, $\mathrm{p}=0.008$ ) and LOS (mean difference -1.28 days $(95 \% \mathrm{Cl}-2.04$ to -0.52$), \mathrm{p}=0.001)$ in favour of $\mathrm{CM}$ compared with usual care. 9 trials described cost data of which 6 reported no difference between CM and usual care. There were 4 studies of communityinitiated CM versus usual care (2 RCTs and 2 NRCTs) with only the 2 NRCTs showing a reduction in admissions.

Conclusions: Hospital-initiated CM can be successful in reducing unplanned hospital readmissions for $\mathrm{HF}$ and length of hospital stay for people with HF. 9 trials described cost data; no clear difference emerged between CM and usual care. There was limited evidence for communityinitiated $\mathrm{CM}$ which suggested it does not reduce admission.

\section{Strengths and limitations of this study}

- High-quality systematic review.

- Interventions examine nurse-led multicomponent care of patients with heart failure.

- Focus on use of resources specific to heart failure.

- Community-initiated case management trials were limited in quantity and were mostly of low quality. - Lack of cost data in most trials.

\section{INTRODUCTION}

Applying current prevalence figures to population estimates suggests that more than 550000 individuals (more than 308000 men and slightly fewer than 250000 women) in the UK are living with heart failure $(\mathrm{HF}){ }^{1}$ Quality and Outcome Framework (QOF) data supports this: in 2012/2013, just over 480000 patients were recorded as having $\mathrm{HF}^{2}$ The average age of patients with $\mathrm{HF}$ in general practice in the UK is 77 years. ${ }^{3}$

Prior to $1990,60-70 \%$ of patients died within 5 years of diagnosis, and admission to hospital with worsening symptoms was a regular and recurrent event. ${ }^{4-6}$ Effective treatment has improved care, with a relative reduction in hospitalisation in recent years of $30-50 \%$, and smaller but significant decreases in mortality. ${ }^{4-6}$

More than $£ 6.8$ billion was spent on treating all cardiovascular disease within the National Health Service (NHS) in England in 2012/ 2013 with $63 \%$ of these costs coming from within secondary care and $21 \%$ within primary care. Within secondary care, non-elective inpatient admittance for cardiovascular disease, that is, emergency admissions, had the greatest expenditure with $£ 1925$ million. ${ }^{1}$ 
Case management $(\mathrm{CM})$ is the process of planning, coordinating and reviewing the care of an individual. We used the definition cited by the King's Fund in the UK 'A collaborative process of assessment, planning, facilitation, care coordination, evaluation, and advocacy for options and services to meet an individual's and family's comprehensive health needs through communication and available resources to promote quality cost-effective outcomes'. ${ }^{7}$ The NHS has used less-intensive approaches than the traditional US model, for example, through the use of nurses to support older people and those with long-term conditions at home. ${ }^{8}$ In this review, we have focused on CM based on nurse coordinated multicomponent care of patients which is applicable to the primary care-based health systems such as that in the UK.

A previous systematic review and meta-analysis shows that $\mathrm{CM}$ is not effective in reducing unplanned hospital admissions for the general older/elderly population. ${ }^{9}$ However, limited data suggest that CM for patients with HF is promising. ${ }^{10}$ This current review aimed to (1) identify the evidence of the effectiveness and related costs of CM interventions for patients with HF predominantly based in the community and (2) to better understand the potential success of $\mathrm{CM}$ by examining the components of tested interventions.

\section{METHODS}

Search

Databases and registries

A search strategy was developed using keywords for the electronic databases according to their specific subject headings or searching structure. The search strategy was run from 1985 to 2012 in the OVID databasesMEDLINE, Excerpta Medica Database (EMBASE), Cumulative Index to Nursing and Allied Health Literature (CINAHL) and PsycINFO on 2 July 2014 (see online supplementary appendix 1 ). The search strategy was modified to search internet sites such as the Agency for Healthcare Research and Quality (AHRQ) and the King's Fund. A pragmatic update of these searches was conducted on 20 November 2015 using the full search strategy and run in MEDLINE and MEDLINE in process only.

\section{Other sources}

Once the included papers were determined, both backwards (reference list of paper) and forwards citation searching (via Google Scholar) was performed to identify any other potentially relevant studies. All authors of included studies in the field were contacted with data queries and to identify additional relevant studies.

\section{Eligibility criteria}

Randomised controlled trials (RCTs) and other controlled studies (non-RCTs, NRCTs; controlled trials, controlled before and after studies, analytic cohorts, comparative studies) were included as determined by our eligibility criteria. We were aware from our previous work that not all community-based studies were randomised and felt it was important to be more inclusive in order to understand why CM may work for HF. CM interventions needed to be initiated either while as an inpatient or on discharge from acute care hospitals including the emergency department (ED), or in the community, and then continue on in the community. Only studies including adults with HF in Organisation for Economic Co-operation and Development (OECD) countries were included as the outcomes were more likely to be comparable for synthesis, and relevant to the UK situation. ${ }^{11}$ Studies were included as long as one of the outcomes of interest was unplanned hospital (re) admissions, ED attendance, length of hospital stay (LOS) as well as related costs of the interventions. Other outcomes of interest were primary healthcare resources, for example, general practitioner visits, visits to other primary care health professionals or services and prescriptions. Studies written in any language were considered if there was an English abstract available.

\section{Reference management and study selection}

EndNote and Excel were used to manage the references. Duplicates were removed from the EndNote file. References underwent a two-stage process of screening using the inclusion and exclusion criteria by two reviewers independently (ALH, AK, RJ). First, a screen of titles and abstracts (if abstract available) and second screening of the full paper was conducted. Where there was continued disagreement between reviewers about including or excluding a paper, a third reviewer made the final decision (SP or RJ).

In addition to the included quantitative intervention papers, we identified relevant reviews from the search. Any potentially relevant conference proceedings were followed up, first by searching in MEDLINE to see if the study had been published. If the study was not published, the authors were contacted where possible to check if the studies were likely to be published within the work frame of this review.

\section{Data extraction and assessment of risk of bias}

Data were extracted into a custom-designed table which included description of trial type, participants, intervention, controls, outcome measures and results. Based on the Kings Fund definition of CM, we devised taxonomy of intervention components ${ }^{8}$ (table 1). As part of this data extraction process, the intervention and control treatments were also described by their component parts, for example, monitoring signs and symptoms using the framework of the CM definition.

Quantitative data concerning the outcomes of interest were extracted into the Cochrane Revman software. The Cochrane risk of bias tool was used to record trial bias for RCTs and the Effective Practice and Organisation of Care (EPOC) risk of bias tool was used for NRCTs. ${ }^{12}$ These processes were performed by one author and checked by a second (ALH, AK). Any disagreements 


\section{Definition and total prevalence of components of CM interventions}

Number of hospital-initiated

CM vs usual care with

component present

(total studies $=16$ )
Number of community-initiated $\mathrm{CM}$ vs usual care with component present (total studies $=4$ )

Assessment/evaluation

Monitoring signs and symptoms $(n=18)$

Encompasses general care of patients with $\mathrm{CHF}$ which is likely to include establishing a relationship with patient over visits, physical and cardiac status checking, lifestyle assessment, general medication check and screening tests, for example, depression, dementia

Medication review $(\mathrm{n}=8)$

Review and adjustment of medication by experienced case manager (nurse), pharmacist, GP or consultant often using a combination of these health professionals

Assessment of home environment $(n=4)$

Assessment carried out by case manager to identify any issues or potential issues with home environment, for example, stairs

CM meetings/feedback to other HPs $(n=5)$

Planning

Group meetings of health professionals involved in patients with CHF care with the aim of reporting on and planning for patients care

Appointment organisation $(n=2)$

Case manager checking medical appointments, ensuring ability to go, etc

Advance care planning $(n=1)$

Facilitation

Education/self-management $(n=18)$

Educating patients with CHF about their condition, treatment and what to expect. The aim of this is to assist self-management (care with assistance of health professionals) and self-care (patient engaging in activities to promote their health and well-being)

Patient-directed access $(n=6)$

The ability of patients with CHF to initiate care from the case manager or CM service

Referral to...( $n=14)$

When the case manager refers the patient to other health or social care professionals, this can be GP hospita

consultant, social care or tests.

Advocacy for options and services

Equipment $(\mathrm{n}=4)$

Provision of items to assist patient's healthcare such as pill counters, weighing scales and measured water bottles

Physical therapy $(n=1)$

Patient with CHF receiving physical therapy/rehabilitation

Support group $(n=1)$

$\mathrm{CHF}$ attending or being offered the opportunity of a support group.

Other

Family involvement $(n=9)$

When the case manager involves the patient's family in terms of information, education or involvement, for example, 8

goal setting in patients' care or active monitoring

Emotional support $(n=1)$

Case manager providing emotional support to patient with $\mathrm{CHF}$.

$\mathrm{CHF}$, chronic heart failure; CM,case management; GP, general practitioner; HP, health professional.

0 
were resolved by discussion and if necessary with a third author (RJ, SP).

\section{Data analysis}

Trials were divided as previously described by Huntley et $a l^{9}$ into hospital-initiated CM and community-initiated CM. Where there were data from three or more studies, effect sizes were calculated and presented in forest plots as rate ratios ((re)admissions) or mean differences (LOS) using Revman software. If the heterogeneity of the combined data was $>50 \%$, a random-effects model was used for analysis.

We conducted prespecified sensitivity analysis in response to the risk of bias assessment of studies, removing high risk of bias studies as appropriate; the results of both analyses are presented. ${ }^{13}$ We conducted prespecified subgroup analysis to explore the effects of CM duration (3, 6 and 12 months plus) on hospital admission and LOS. There was insufficient detail in trials to perform subanalysis by severity of HF or intensity of intervention.
Data were assessed narratively in respect of the components of interventions using the CM definition cited above as guidance ${ }^{8}$ (table 1 ). In addition, where possible, post hoc subgroup analysis was conducted in Revman in which interventions with components of interest were compared with those that did not have these components.

\section{RESULTS}

The systematic review yielded 22 studies with data published over 32 papers of which 17 were RCTs and 5 were NRCTs, all published in the English language ${ }^{14-45}$ (figure 1). No relevant studies were identified in a pragmatic update using the full search strategy run in MEDLINE and MEDLINE in process only in November 2015. Seventeen of these studies described hospital-initiated CM (n=4794), ${ }^{14} 1517 \quad 18$ 20-24 26-28 3132 and five described community-initiated $\mathrm{CM}$ of $\mathrm{HF}$ $(\mathrm{n}=3832) .{ }^{38}{ }^{42-45}$ The PRISMA checklist was used to ensure the quality of our systematic review manuscript.

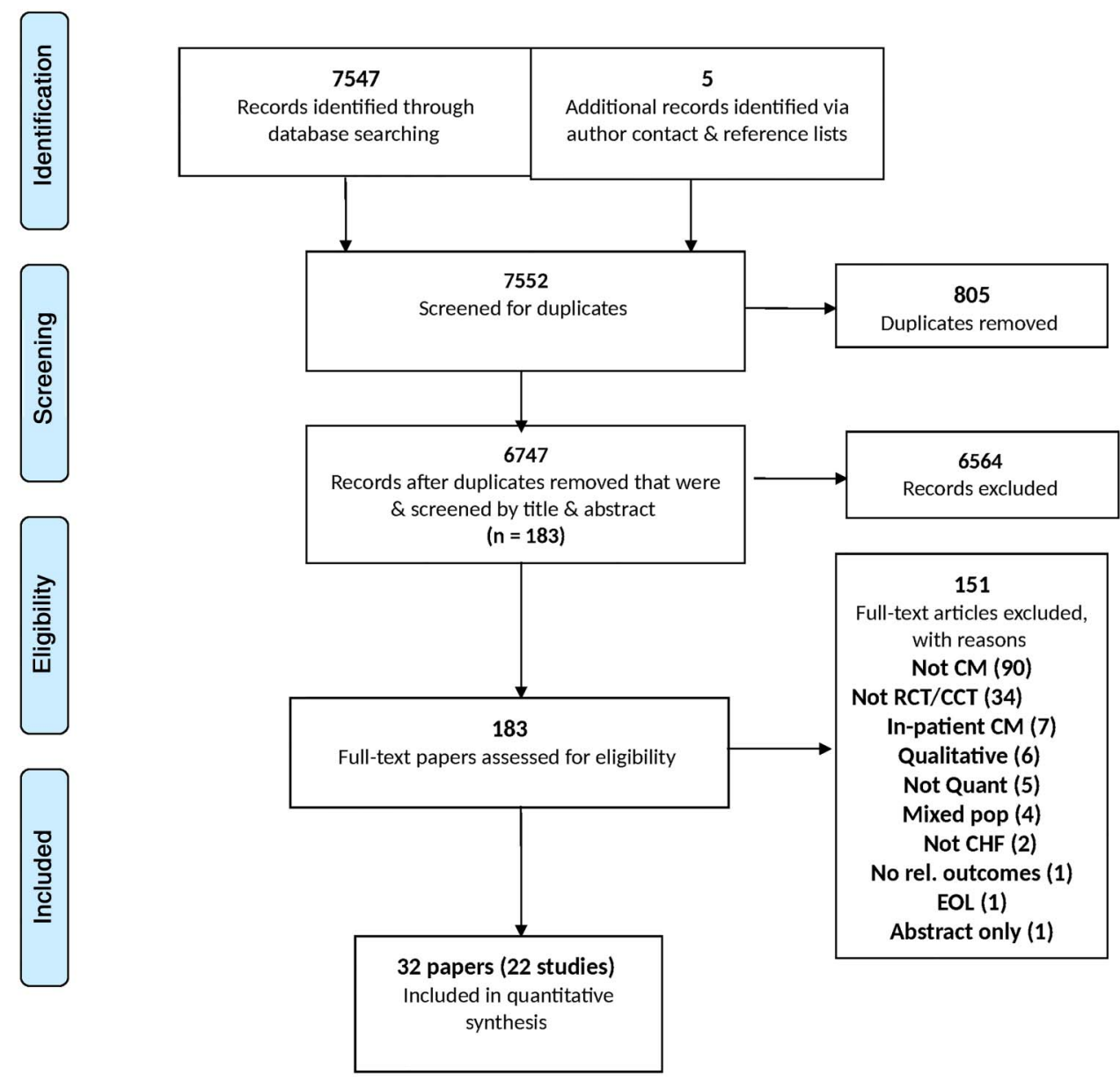

From: Moher D, Liberati A, Tetzlaff J, Altman DG, The PRISMA Group (2009). Preferred Reporting /tems for Systematic Reviews and MetaAnalyses: The PRISMA Statement. PLoS Med 6(6): e1000097. doi:10.1371/journal.pmed1000097

For more information, visit www.prisma-statement.org.

Figure 1 PRISMA flow diagram. CCT, controlled clinical trial; CHF, chronic heart failure; CM, case management; EOL, end of life; RCT, randomised controlled trial. 


\section{Profile of patients}

The range of female participants in the trials was $1-58 \%$, but the majority of trials had relatively even gender divide (table 2). Comorbidity and multimorbidity were common. Eight of the 22 trials gave no detail on ethnicity of participants; in four studies, the triallists used white/non-white and English-speaking/non-Englishspeaking categories. In the remaining 10 studies, a fuller profile was described. Twelve of the 22 trials were conducted in the USA and the ethnicity profile reflected that including Spanish speaking/Hispanic, American Indian, black, African-American, Asian and white participants.

The majority of trials described the severity of $\mathrm{HF}$ using New York Heart Association (NYHA) classification. Twelve of the trials gave a breakdown of numbers or percentages in the I-IV classes with some trials only giving numbers of participants for the III and IV classes. In these trials, the percentage range of III and IV class patients was $6-98 \%$. Four trials gave mean and median values of NYHA status, one trial used the all-patient refined-diagnosis related group (APR-DRG) severity of illness scale, and five trials did not describe disease severity.

\section{Profile of interventions}

The majority of studies $(n=15)$ described the intervention being delivered by a case manager/specialist nurse with no specific mention of other health professionals, and the remaining seven studies described a case manager/specialty nurse working as part of a multidisciplinary team (table 2 ).

All but two studies compared CM with usual care although the control group was not always described. The two remaining studies were comparative: one RCT comparing CM with specialist clinics and one RCT comparing CM with telemedicine plus CM. ${ }^{32} 42$

The duration of the CM interventions in the studies was 1-24 months with the majority having a 3-month or 6 -month duration. The majority of studies were conducted face to face or a combination of in-person and by phone. Four interventions were conducted purely by phone. $^{21} 222642$ Outcomes were measured to match the total duration of intervention in the majority of studies. For many of the studies, the intensity of interventions was not stated explicitly. When intensity was described, it was always a tapered approach after an initial intensive period.

\section{Risk of bias}

The degree of risk of bias was starkly different between the RCTs and NRCTs. All five of the NRCTs were rated at high risk or unknown risk for most domains (figure 2A, B). ${ }^{35-37} 4445$

The majority of the RCTs were rated at low risk for most domains with the exception of the domain of blinding of the participants and personnel which is not applicable to this type of intervention. Three RCTs were assessed as at high risk for at least one domain: both Hancock $e t a l^{43}$ and Wade $e t a l^{42}$ gave no description of the randomisation process or allocation concealment, Riegel $e t a l^{21}$ was randomised at physician level and patients were chosen by physician preference. Four of the five community-initiated trials (two RCTs and two NRCTs) were assessed to be at high risk of bias, and in some studies did not present usable data. ${ }^{35-37} 42$

All the intervention studies reported unplanned hospital (re)admissions ${ }^{14-45}$ and 17 reported length of time in hospital. ${ }^{14} 151718$ 20-22 24 26-28 3538 42-45 There were few data on $\mathrm{A}$ and $\mathrm{E}$ attendance and primary care resource use. However, only some of the data could be used in meta-analysis with the main reasons being that data were presented in different formats where neither CIs, SEs nor raw data were given. Owing to heterogeneity of data, all analyses were conducted using a random-effects model.

\section{Unplanned HF (re)admissions data \\ Hospital-initiated CM}

Thirteen of the hospital-initiated CM trials had data that could be used in a meta-analysis of which 12 were RCTs. The pooled data from the RCTs showed a rate ratio of readmissions of 0.74 (95\% CI 0.60 to $0.92 ; \mathrm{p}=0.008$; $\mathrm{I}^{2}=69 \%$ ) in favour of hospital-initiated CM (figure 3A). A sensitivity analysis was conducted, removing Riegel et $a l^{21}$ (RCT with high risk of bias for randomisation domain); this had a minimal effect on the rate ratio and heterogeneity $(0.77$ (0.61 to 0.96$\left.) ; \mathrm{p}=0.02 ; \mathrm{I}^{2}=68 \%\right) .^{35}$ Subanalysis looking at 3-month, 6-month and 12-18-month data did not produce a clear time-related effect which is most likely due to heterogeneity within and between studies (figure 3B-D). There was one hospital-initiated CM trial which compared CM with specialist clinics which reported no differences in hospital readmissions between the two groups. ${ }^{32}$

\section{Community-initiated CM}

Of the four community-initiated trials (two RCTs and two NRCTs) comparing admissions between CM with usual care, two reported no significant differences ${ }^{38} 43$ and two reported statistically significant reductions in favour of CM. ${ }^{444}$ One further trial compared CM, with telehealth and $\mathrm{CM}$ and reported no differences in admissions but data were not presented. ${ }^{42}$

\section{Length of hospital stay}

\section{Hospital-initiated CM}

Nine of the hospital-initiated CM trials had data that could be used in a meta-analysis of which eight were RCTs. The pooled data from the RCTs showed that mean LOS was reduced in the CM group compared with usual care mean difference (MD -1.28 days $(-2.04$ to -0.52$)$; $\mathrm{p}=0.001 ; \mathrm{I}^{2}=63 \%$; figure $\left.4 \mathrm{~A}\right)$. A sensitivity analysis was conducted removing Riegel $e t a l^{21}$ which had an important effect on the rate ratio and heterogeneity (MD -1.76 $(-2.29$ to -1.23$\left.) ; \mathrm{p}<0.00001 ; \mathrm{I}^{2}=14 \%\right) .{ }^{21} 35$ Subanalysis looking at 3-month, 6-month and 12-18-month data suggests this effect is short term (first 3 months) but a 
Table 2 Study characteristics of intervention studies

\section{Study \\ $\mathrm{n}=$ randomised \\ Recruitment/setting \\ Baseline characteristics of \\ participants: CM vs usual \\ care}

Hospital-initiated CM-RCTs

Rich et al, ${ }^{14}$ USA

$\mathrm{n}=98$ randomised

Patients $\geq 70$ years admitted to medica

wards of Jewish Hospital at Washington

University Medical Centre were screened

for congestive HF.

Rich et al, ${ }^{15}$ USA

$\mathrm{n}=285$ randomised

As above for Rich et al $^{14}$

\section{Stewart et $a l,{ }^{17}$ Australia}

$\mathrm{n}=97$ randomised

Patients were recruited while admitted to a

large tertiary hospital

Stewart et al, ${ }^{18}{ }^{19}$ Australia

$\mathrm{n}=200$ randomised

Patients admitted to a tertiary referral

hospital

Female (\%): $60.3,47.1 \%$

Female (\%): 55, 48\%
Intervention

Age: 80 (6.3), 77.3 (6.1)

years $\mathrm{p}=0.04$

Ethnicity: white $46,57.1 \%$

Disease status:

Mean NYHA status

2.7 (1.1), 3.0 (1.0)

Age: 80.1 (5.9), 78.4 (6.1)

years $\mathrm{p}=0.02$

Female (\%): 68, 59\%

Ethnicity: non-white race 52

$59 \%$

Disease status:

Mean NYHA class

2.4 (1.0), 2.4 (1.1)

Age: 76 (11), 74 (10) years

Ethnicity: non-English

speaking

$20.4,18.75 \%$

Disease status: NYHA

II $49,50 \%$

III $47,42 \%$

III $4,4 \%$

Age: 75.2 (7.1), 76.1 (9.3)

Female (\%): 35, 41\%

Ethnicity: primary language

not English 32, 32

Disease status: NYHA

II 42,48

III 46,43

IV 12,9
Non-pharmacological

comprehensive multidisciplinary

treatment strategy NPCM $(\mathrm{n}=63)$

Components of intervention at

home:

Monitoring signs and symptoms

Medication review (nurse)

Education/self-management

support

Assessment of home environment

Patient-directed access to study personnel

Nurse-directed multidisciplinary

intervention $(\mathrm{n}=142)$

As above for Rich et al $^{14}$

Components of intervention at

home:

Monitoring signs and symptoms

Education (pharmacist)/

self-management support

Medication review (pharmacist)

Referral to GP

Family involvement

Equipment

Multidisciplinary home base

intervention $(n=100)$

Components of intervention at

home:

Monitoring signs and symptoms

Referral to other health and social

care

Appointment organisation
Contro

Main results

Intervention vs contro

UC $(\mathrm{n}=35)$

Components of $\quad 21(\mathrm{Cl} 21.7 \%$ to $44.9 \%)(33.3 \%)$

intervention: visits by $16(29.2 \%$ to $62.2 \%)(45.7 \%)$

home nurse

Total hospital days: 272,200

Mean number of days: 4.3

(SD1.1), 5.7 (SD2.0)
UC $(\mathrm{n}=140)$

As above for Rich

et $a l^{14}$

UC $(\mathrm{n}=48)$

Components

intervention: DM
Number of readmissions

24, $54 \mathrm{p}=0.04$

Total hospital days

556, 865

Mean number of days

3.9 (10), 6.2 (11.4)

$\mathrm{p}=0.04$

Number of readmissions

36, $63(\mathrm{p}=0.03)$

Number of patients experiencing

a readmission

$24,31(\mathrm{p}=0.12)$

LOS in days

$261,452(p=0.05)$

UC $(n=100)$

Components of

intervention:

Contact with other

health and social

professionals

Appointment with GP

or cardiac clinic or both 
Table 2 Continued

\begin{tabular}{ll}
\hline Study & Baseline characteristics of \\
$\mathrm{n}=$ randomised & participants: CM vs usual \\
Recruitment/setting & care
\end{tabular}

care

Intervention

Assessment of home environment

Family involvement

Education/self-managemen

support

Medication review (nurse/GP

cardiologist)

Blue et $a l^{20}$ UK

$\mathrm{n}=165$ randomised

Age: (SD) 74.4 (8.6), 75.6

(7.9) years

Patients admitted as an emergency to the Female (\%): $36,49 \%$

acute medical ward of the hospital

Riegel et al, ${ }^{21}$ USA

Ethnicity: not reported

Disease status: NYHA

II 19 (23), 16 (20)

III 28 (34), 33 (42)

IV 36 (43), 30 (38)

Comorbidity or

multimorbidity:

Angina 40 (49), 38 (45)

Past MI 41 (51), 46 (55)

Diabetes 15 (19), 15 (18)

Chronic lung disease 18

(22), 23 (27)

Hypertension 42 (52), 36

(43)

AF 42 (52), 29 (35)

Valve disease 12 (15), 15

(18)

$\mathrm{n}=281$ physicians randomised

Age: 72.52 (13.05), 74.63

(12)

Patients admitted at 2 Southern California

hospitals

Female (\%): 46.2, 53.9

Ethnicity: (primary language) Monitoring signs and symptoms

English 91 (70), 168 (73.7)

Spanish 35 (26.9), 58 (25.4)

Disease status: NYHA

II 2.3, 3.6

III $35.9,38.4$

IV $61.7,58.0$
Specialist nurse intervention $(\mathrm{n}=82)$

Components of intervention at

home:

Monitoring signs and symptoms

Education/self-management

support

Referral to other health and social

care

Appointment organisation

Medication review (nurse,

cardiologist )

\section{Telephonic CM $(\mathrm{n}=130)$}

Components of intervention at

Self-management support

Referral to other HPs (including

Family involvement
GP) and social care

Control

Main results

Intervention vs control

$p=0.004$

18 months

Number of readmissions

$64,125, \mathrm{p}=0.02$

Mean number of hospital days

10.5 (14.4), 21.1 (24.1) days per patient, $\mathrm{p}=0.004$

UC $(\mathrm{n}=75)$

Components of

Number(\%) of readmissions

12 (14), 26 (32)

intervention: GP care $\operatorname{HR} 0.38(0.19,0.76) \mathrm{p}=0.0044$

LOS in days

3.43 (12.2), $7.46(16.6)$

Cl 0.6 (0.41 to 0.88$), p=0.0051$

UC $(\mathrm{n}=228)$

Components of

intervention: not

known
Readmission rates

3 months $14.6,22.8 \mathrm{p}=0.06$

(calculation 19 people vs 52 experiencing 1 or more admission)

6 months 17.7, $27.6 \mathrm{p}=0.06$

(calculation 23 people vs 63 people experiencing 1 or more admision)

LOS in days

3 months 0.85 (2.3), 1.6 (3.9)

$\mathrm{p}=0.56$

6 months 1.1 (3.1), 2.1 (4.6)

$\mathrm{p}=0.05$ 


\begin{tabular}{|c|c|c|c|c|}
\hline $\begin{array}{l}\text { Study } \\
\mathrm{n}=\text { randomised } \\
\text { Recruitment/setting }\end{array}$ & $\begin{array}{l}\text { Baseline characteristics of } \\
\text { participants: CM vs usual } \\
\text { care }\end{array}$ & Intervention & Control & $\begin{array}{l}\text { Main results } \\
\text { Intervention vs control }\end{array}$ \\
\hline $\begin{array}{l}\text { Laramee et al, }{ }^{22} \text { USA } \\
\mathrm{n}=287 \text { randomised } \\
\text { Patients admitted to hospital for CHF were } \\
\text { screened. }\end{array}$ & $\begin{array}{l}\text { Age: } 70.6(11.4), 70.8(12.2) \\
\text { years } \\
\text { Female (\%): } 42,50 \% \\
\text { Ethnicity: not reported } \\
\text { Disease status (SD): } \\
\text { NYHA } \\
\text { I } 10(7), 35(26) \\
\text { II } 76(55), 47(36) \\
\text { III } 50(36), 46(35) \\
\text { IV } 3(2), 4(3) \\
\text { Note } p<0.001\end{array}$ & $\begin{array}{l}\text { CM ( } \mathrm{n}=131 \text { data available ) } \\
\text { Components of intervention at } \\
\text { home: } \\
\text { Monitoring signs and symptoms } \\
\text { Education/self-management } \\
\text { support } \\
\text { Family involvement } \\
\text { Equipment } \\
\text { Patient-directed access to CM }\end{array}$ & $\begin{array}{l}\text { UC }(\mathrm{n}=125 \text { data } \\
\text { available) } \\
\text { Components of } \\
\text { intervention: not } \\
\text { known }\end{array}$ & $\begin{array}{l}\text { Number of readmissions } \\
3 \text { months period } \\
18(14) \text { vs } 21 \text { (17) NS } \\
\text { LOS in days } \\
\text { in hospital for those patients with } \\
\geq 1 \text { readmission } \\
6.9(6.5), 9.5(9.8) \mathrm{NS}\end{array}$ \\
\hline $\begin{array}{l}\text { DeBusk et } a l^{23} \text { USA } \\
\mathrm{n}=462 \text { randomised } \\
\text { Patients who were admitted with a } \\
\text { provisional diagnosis of HF from Kaiser } \\
\text { Permanente medical centres in California }\end{array}$ & $\begin{array}{l}\text { Age: }<60 \text { years } 15,14 \%, 6- \\
70 \text { years } 22,24 \%, 70- \\
80 \text { years } 40,37 \%,>80 \text { years } \\
21,26 \% \\
\text { Female (\%): } 52,45 \% \\
\text { Ethnicity: American Indian 0, } \\
1 \% \\
\text { Asian } 4,8 \% \\
\text { Black } 2,2 \% \\
\text { White } 5,6 \% \\
\text { Hispanic } 3,3 \% \\
\text { Disease status: NHYA } \\
\text { I-II } 50,50 \% \\
\text { III-IV } 50,50 \%\end{array}$ & $\begin{array}{l}\text { CM }(\mathrm{n}=228) \\
\text { Components of intervention at } \\
\text { home: } \\
\text { Monitoring signs and symptoms } \\
\text { Education/self-management } \\
\text { support } \\
\text { CM meetings/feedback to other } \\
\text { health providers }\end{array}$ & $\begin{array}{l}\text { UC }(\mathrm{n}=234) \\
\text { Components of } \\
\text { interventions: not } \\
\text { known }\end{array}$ & $\begin{array}{l}\text { Total number of readmissions } \\
\text { in } 1 \text { year } \\
76,86 \text { no stats available }\end{array}$ \\
\hline $\begin{array}{l}\text { Naylor et al, }{ }^{24} 25 \text { USA } \\
n=239 \text { patients randomised } \\
\text { Patients aged } 65 \text { years+ admitted to } 6 \\
\text { study hospitals from home with a } \\
\text { diagnosis of HF were screened for } \\
\text { participation. }\end{array}$ & $\begin{array}{l}\text { Age: } 76.4(6.9), 75.6(6.5) \\
\text { Female (\%): } 60,56 \% \\
\text { Ethnicity: African-American } \\
34,38 \% \text {, white } 66,62 \% \\
\text { Disease status: } \\
\text { Functional status (Moinpur C } \\
\text { 1992) } \\
\text { Personal } 17.1(5.8), 16.9 \\
\text { (5.8) } \\
\text { Social } 8.4(2.6), 8.6(2.6) \\
\text { Total } 25.5(8), 25.4(7.8)\end{array}$ & $\begin{array}{l}\text { Transitional care intervention with } \\
\text { APNs }(\mathrm{n}=118) \\
\text { Components of intervention at } \\
\text { home: } \\
\text { Monitoring signs and symptoms } \\
\text { Education/self-management } \\
\text { support } \\
\text { Family involvement } \\
\text { CM meetings/feedback to other } \\
\text { health providers } \\
\text { Patient-directed access to } \mathrm{CM}\end{array}$ & $\begin{array}{l}\text { UC }(\mathrm{n}=121) \\
\text { Components of } \\
\text { intervention: } \\
\text { Care from standard } \\
\text { home care services } \\
\text { Patient-directed } \\
\text { access to home care } \\
\text { services }\end{array}$ & $\begin{array}{l}\text { Number of readmissions } \\
40 \text { vears } 72 \mathrm{NS} \\
\$ 175840, \$ 498110 \\
\text { Total hospital days (all cause) } \\
588,970 \\
\text { Per patient, mean } \pm \text { SD } 5.0 \pm 7.3 \\
8.0 \pm 2.3 \text { NS } \\
\text { Per hospitalised patient, mean } \\
\pm \text { SD } \\
11.1 \pm 7.214 .5 \pm 13.4 \mathrm{NS}\end{array}$ \\
\hline $\begin{array}{l}\text { Riegel et al, }{ }^{26} \text { San Diego, USA } \\
n=135 \text { randomised }\end{array}$ & $\begin{array}{l}\text { Age: } 71.6910 .8), 72.7(11.2) \\
\text { Female (\%): 58, } 49.2 \%\end{array}$ & $\begin{array}{l}\text { Telephonic CM }(\mathrm{n}=69) \\
\text { Components of intervention at }\end{array}$ & $\begin{array}{l}\text { Usual care }(\mathrm{n}=65) \\
\text { Components of }\end{array}$ & $\begin{array}{l}\text { Readmission rates } \\
\text { (\%) (number of people) all NS }\end{array}$ \\
\hline
\end{tabular}




\section{Study \\ $\mathrm{n}=$ randomised \\ Baseline characteristics of \\ participants: CM vs usual}

Recruitment/setting

care

Intervention

Self-identified Hispanics were identified at Ethnicity: Hispanic patients

2 community hospitals close to

US-Mexico border.

Speak/read only Spanish

$60.9,65.1 \%$

Disease Status: NYHA

II $17.4,20 \%$

III $44.9,47.7 \%$

IV $37.7,32.3 \%$

Age: 73 (14), 72 (12)

Thompson et al, ${ }^{27}$ UK

Randomisation was at GP practice level

Patients recruited from 2 North of England

general hospitals following an admission

Female (\%):38, 27\%

Ethnicity: no details

Disease status:

NYHA III and IV

$76,73 \%$

Jaarsma et $a l,{ }^{28} 29$ The Netherlands $\mathrm{n}=1049$ randomised

Age: 71 (11), 70 (12), 72 (11)

All patients had been admitted to hospital Female (\%):34, 39, 40\% with symptoms of $\mathrm{HF}$.

\section{home:}

Monitoring signs and symptoms

self-management support

Referral to other HPs (including

GP) and social care

Family involvement

Clinic and home-based intervention $(\mathrm{n}=58)$

Components of intervention at

home:

Monitoring signs and symptoms

Education/self-management

support

Family involvement

In outpatient clinic

Monitoring signs and symptoms

Education/self-management

support

Family involvement

Referral to other health and social

care

BNS $(\mathrm{n}=340)$

Components of intervention:

Outpatients

Education/self-management

support

Patient directed access to HF nurse

INS ( $\mathrm{n}=344)$

Components of intervention at

home:

Patient-directed access to HF nurse

Referral to other health and socia

care

Education/self-management

Control

Main results

Intervention vs control

intervention: DM

months $8.7,13.8 \%$

information

(Calculation 6.003, 8.97)

3-month 21.7, 26.2\%

(Calculation 14.49, 17.03)

6 months $31.9,33.8 \%$

(Calculation 22.011, 21.97)

LOS in days

(mean) all NS

1 months 0.59 (2.3), 1.41 (5.5)

3 months 2.19 (5.4), $2.4(6.2)$

6 months 3.65 (7.8), 3.4 (7.1)

Number of patients experiencing

one or more readmissions

Components of

intervention at home: 13,21

unknown

Total number of readmissions

15, 45

Total number of hospital days

108, 459

$p<0.01$ for all at 6 months

UC group ( $n=339)$

Components of

intervention: DM
Number of readmissions $121,134,120 \mathrm{NS}$

LOS in days

(medians)

$8.0(4,14), 9.5(5,17), 12$

$(5,19.5)$

$p<0.01$ between BNS group and control but NS between INS

group and control 


\section{Study}

$\mathrm{n}=$ randomised

Recruitment/setting

Brotons et al, ${ }^{31}$ Spain

$\mathrm{n}=283$ randomised

Patients were recruited by well-trained

nurses at 2 university hospitals.

Stewart et al, ${ }^{32} \mathrm{WHICH}$ trial, Australia $\mathrm{n}=280$ randomised

Patients admitted to participating hospitals were screened for study eligibility.

Hospital-Initiated CM-NRCTs

Riegel et al, ${ }^{35}$ USA

$\mathrm{n}=240$ were randomised

Patients were recruited from 5 hospitals

following a hospitalisation for HF.

$\mathrm{n}=447$
Baseline characteristics of

participants: CM vs usual

care

Intervention

support

Equipment

Age: 76.6 (7.5), 76 (8.9)

years.

Female (\%): 54.2, 56.1\%

Ethnicity: not reported

Disease status: NHYA

I 42.4, 55.4\%

II $52.1,37.4 \%$

III $4.9,5.8 \%$

IV $0.7,1.4 \%$

Home vs clinic

Age: 70 (15), 73 (13) years

Female (\%):27, 28\%

Ethnicity: no details

Disease status: NYHA II or

III

$83,88 \%$

Home-based intervention $(n=144)$

Components of intervention at

home:

Monitoring signs and symptoms

Education/self-management

support

Medication review (nurse,

physician, cardiologist)

Referral to physician or cardiologist

as necessary

Home-based intervention $(n=143)$

Components of intervention at

home:

Monitoring signs and symptoms

Family involvement

$\mathrm{CM}$ meetings/feedback to other

health providers

Months since CHF diagnosis Referral to other health or social 34.6 (55.3), 44.8 (71.0)

care

Assessment of home environment

Medication review (nurse,

pharmacist, physician, cardiologist)

Age: 74.44 years. (10.65),

70.77 (11.77)

Female (\%): 55, 55\%

Ethnicity: no details

Disease status: NYHA

I $19.2,24.2 \%$

II $26.7,18.3 \%$

III $43.3,44.2 \%$

IV $10.8,13.3 \%$

Age: 79.4 (10.7), 79.9 (10.7)

Female (\%): 55.6, 57.6

(numbers)
Transitional care service $(n=223)$

Components of intervention at home:
Multidisciplinary DM $(\mathrm{n}=120)$

Components of intervention at

home:

Monitoring signs and symptoms

Support group

Referral to specialist $\mathrm{RN}$ visits

Control

Main results

Intervention vs control

UC $(\mathrm{n}=139)$

Components of intervention: not

known

Clinic-based intervention $(\mathrm{n}=137)$

Components of intervention:

In clinic

DM

Assessment of home

environment

Family involvement?

Referral to other

health or social care

CM meetings/

feedback to other

health providers

UC $(\mathrm{n}=120)$

Components of

intervention at home:

DM

3 months

$0.22(0.52), 0.13(0.45)$ (NS)

6 months

0.32 (0.58), 0.23 (0.53) (NS)

LOS in days

3 months

0.89 (3.34), 0.48 (1.64) (NS)

6 months

1.31 (3.77), 1.08 (3.46) (NS)

Usual home care

services $(\mathrm{n}=224)$

Components of
Readmissions

Unadjusted OR 30 days $0.58(0.38,0.88) \mathrm{p}<0.01$ 


\begin{tabular}{|c|c|c|c|c|}
\hline $\begin{array}{l}\text { Study } \\
\text { n=randomised } \\
\text { Recruitment/setting }\end{array}$ & $\begin{array}{l}\text { Baseline characteristics of } \\
\text { participants: CM vs usual } \\
\text { care }\end{array}$ & Intervention & Control & $\begin{array}{l}\text { Main results } \\
\text { Intervention vs control }\end{array}$ \\
\hline $\begin{array}{l}\text { Patients were referred from a single large } \\
\text { not-for-profit general medical and surgical } \\
\text { hospital. }\end{array}$ & $\begin{array}{l}\text { Ethnicity: } \\
\text { White non-Hispanic } 56.9, \\
58.4 \\
\text { African-American } 17.0,16.5 \\
\text { Hispanic } 14.8,14 . \\
\text { Asian/other } 11.2,10.7 \\
\text { Disease status: patients with } \\
\text { a primary or secondary } \\
\text { diagnosis of CHF }\end{array}$ & $\begin{array}{l}\text { Self-management support } \\
\text { Referral to other health and social } \\
\text { care } \\
\text { Assessment of home environment } \\
\text { CM meetings/feedback to other } \\
\text { health providers } \\
\text { Advance care planning } \\
\text { Physical therapy }\end{array}$ & $\begin{array}{l}\text { intervention at home: } \\
\text { Nurse visits } \\
\text { Physical therapy } \\
\text { (44.6) } \\
\text { Home health aide } \\
\text { service (27.7) }\end{array}$ & $\begin{array}{l}\text { Adjusted OR } 30 \text { days } \\
0.57(0.38,0.87) p<0.01\end{array}$ \\
\hline $\begin{array}{l}\text { Stauffer et al, }{ }^{37} \text { USA } \\
\mathrm{n}=140 \\
\text { Patients were screened for eligibility within } \\
48 \mathrm{~h} \text { of hospital admission }\end{array}$ & $\begin{array}{l}\text { Age: } 78.9(8.3), 81.4(8.3) \\
\text { Female (\%): } 58.1,54.8 \% \\
\text { Ethnicity: Hispanic ethnicity } \\
7.1,3.6 \% \\
\text { Disease status: } \\
\text { APR-DRG severity of illness } \\
15.4,1.2 \% \\
244.6,31 \% \\
337.5,57.1 \% \\
412.5,10.7 \%\end{array}$ & $\begin{array}{l}\text { Nurse-led transitional care } \\
\text { intervention ( } \mathrm{n}=56 \text { ) } \\
\text { Components of intervention at } \\
\text { home: } \\
\text { Monitoring signs and symptoms } \\
\text { Education/self-management } \\
\text { support } \\
\text { Family involvement } \\
\text { Referral (assessing availability of } \\
\text { social care) } \\
\text { Patient-directed access to study } \\
\text { personnel }\end{array}$ & $\begin{array}{l}\text { Control group }(\mathrm{n}=84) \\
\text { Components of } \\
\text { interventions: } \\
\text { unknown }\end{array}$ & $\begin{array}{l}\text { Readmission rate at } 30 \text { days } \\
12.6(7.4,17.8) \text { difference }-12.6 \text {, } \\
\text { per cent change }-48 \% ; 16.4(14 \text {, } \\
18.7) \text { difference }-1.6 \% \text { change } \\
11 \%\end{array}$ \\
\hline \multicolumn{5}{|l|}{ Community-initiated $C M-R C T s$} \\
\hline $\begin{array}{l}\text { Peters-Klimm et al, }{ }^{39} \text { Germany } \\
\mathrm{n}=199 \text { at randomisation } \\
\text { Recruitment was via general practice by } \\
\text { mail. }\end{array}$ & $\begin{array}{l}\text { Baseline characteristics of } \\
\text { participants: CM, UC } \\
\text { Age: } 70.4 \text { years (10.0), } 68.9 \\
\text { (9.7) } \\
\text { Female (\%):29, } 26 \% \\
\text { Ethnicity: no details } \\
\text { Disease status: NYHA } \\
\text { I } 1(1.0), 5(5) \\
\text { II } 63(64.9), 67(67) \\
\text { III } 33(34), 27(27) \\
\text { IV 0, } 1(1.0) \\
\text { Mean years with CHD } \\
6.2(4.6)(n=79), 6.8(6.3) \\
(n=74)\end{array}$ & $\begin{array}{l}C M(\mathrm{n}=97) \\
\text { Components of intervention at } \\
\text { home: } \\
\text { Monitoring signs and symptoms } \\
\text { Education/self-management } \\
\text { support } \\
\text { Medication review (CM/GP) } \\
\text { Referral to GP }\end{array}$ & $\begin{array}{l}\text { UC }(\mathrm{n}=100) \\
\text { Components of } \\
\text { control intervention: } \\
\text { DM } \\
\text { Education }\end{array}$ & $\begin{array}{l}\text { Number of admissions } \\
\text { (baseline } 36 \text { vs } 35 \text { ) } \\
18 \text { vs } 9 \text { at } 12 \text { months (NS) } \\
\text { Number of patients experiencing } \\
\text { one or more CHF admissions } \\
11 \text { vs } 7 \text { at } 12 \text { months (NS) }\end{array}$ \\
\hline $\begin{array}{l}\text { Wade et } a l^{42} \text { USA } \\
\mathrm{n}=2200 \text { were randomised }\end{array}$ & $\begin{array}{l}\text { Age: } 75.8,77.7 \text { years. } \\
\text { Female (\%): no detail }\end{array}$ & $\begin{array}{l}C M(n=152) \\
\text { Components of intervention at } \\
\text { home: }\end{array}$ & $\begin{array}{l}\text { THCM }(n=164) \\
\text { Components of } \\
\text { intervention: }\end{array}$ & $\begin{array}{l}\text { No data available for primary } \\
\text { outcome but described as NS }\end{array}$ \\
\hline
\end{tabular}

Recruitment/setting

Patients were referred from a single large not-for-profit general medical and surgical

Patients were screened for eligibility within

$48 \mathrm{~h}$ of hospital admission home: 


\section{Study}

n=randomised

Recruitment/setting

Aetna Medicare Advantage members with medical and pharmacy benefits were identified through analysis of claims.

Hancock et $a l,{ }^{43}$ UK

$\mathrm{n}=28$ randomised

Residents from 33 of 35 long-term

residential and nursing homes
Baseline characteristics of

Community-initiated $\mathrm{CM}-\mathrm{NRCTS}$

Bonarek-Hessamfar et al, ${ }^{44}$ France

$\mathrm{n}=362$

Compared patients included prospectively from 1 January 2004 to 31 December

2005 from GP list
Age: median 78,80 years. Female (\%): no details

Ethnicity: no details

Disease status: NHYA

Median of III, IV
Lowery et al, ${ }^{45}$ USA

$\mathrm{n}=1043$

Intervention implemented in 4 Midwest VA medical centres from the same region and

one affiliated outpatient clinic and 2 VA

medical centres served as control. participants: CM vs usual

care

Ethnicity: black/

African-American

$24,20.4 \%$

Disease status (SD): no

detail

Age: 85.1 (6.7), 81.8 (7.1)

years

Female (\%):56\%, 58\%

Ethnicity: $100 \%$ white British

Disease status:

I:II:III:IV

10:1:4:1, 5:4:1:1

\section{Intervention Control}

Referral to other health and social DM

care

Equipment

\section{$C M(\mathrm{n}=16)$}

Components of interventions at home:

Monitoring signs and symptoms

Education

CM meetings/feedback to other

health providers

Medication review (CM/GP/

cardiologist )

\section{Coordinated care via}

multidisciplinary network $(n=129)$

Components of intervention at

home:

Monitoring signs and symptoms

Education (diet)

Physical therapy

CM meetings/feedback to other

health provider

Age: 65.4 (0.51), 67.4 (0.45) Nurse-practitioner-led DM model

years.

Female (\%):1, $1 \%$

Ethnicity:

White $71.2,79.9 \%$

Black 24, 16.1\%

Other $4.8,4.0 \%$

Disease status: no details

\section{$(\mathrm{n}=457)$}

Components of intervention at

home:

Location was lead tertiary centre, other medical centres (some primary care) or one affiliated outpatient clinic.

Monitoring signs and symptoms

Education/self-management

support

Referral to other health and socia

care

Family involvement

\section{Intervention vs control}

The participant population overal had $42 \%$ fewer inpatient days during the intervention period compared with the previous year. No data

Referral to oth health and socia care

Routine GP-led care

$(\mathrm{n}=12)$

Components of

intervention: DM

mber of admissions

at 6 and 12 months

0,0 at 6 months

0,0 at 12 months
UC $(\mathrm{n}=233)$

Components of intervention: not known

UC $(\mathrm{n}=510)$

Components of intervention: not known
Number of patients experiencing at least one admission

26, 58

Total number of admissions

35, 96

Median LOS

9.2, 11.7 days

In the 2-year period

Mean number of readmissions 1 year

0.7 (0.32), $0.23(0.65) p<0.001$

$(417,428)$

2-year

0.15 (0.58), $0.13(0.42) \mathrm{NS}$

$(384,382)$

Mean number of days in hospital 1 year

0.37 (2.25), 0.97 (3.15) $\mathrm{p}=0.0014$

2-year

0.86 (3.98), 0.66 (2.74) NS

AF, atrial fibrillation; APN, advanced practice nurse; APR-DRG, all-patient refined-diagnosis related group; BNS, basic nurse support; CHD, coronary heart disease; CHF, chronic heart failure; CM, case management or case manager: COPD chronic obstructive pulmonary disease; DM disease management; GP general practitioner; HOCM/RCM, hypertrophic obstructive/restrictive cardiomyopathy; HP, health professional; INS, intensive nurse support; LOS, length of hospital stay; LV, left ventricular; MI, myocardial infarction; NPCM, non-pharmacological comprehensive cardiomyopathy; HP, health professional; INS, intensive nurse support; LOS, length of hospital stay; LV, left ventricular; MI, myocardial infarction; NPCM, non-pharmacological comprehensive
multi-disciplinary treatment strategy; NRCT, non-randomised controlled trial; NS, not statistically significant; NYHA, New York Heart Association; PAD, peripheral arterial disease; SNF, skilled nursing facility; THCM, telehealth with CM; UC, usual care. 


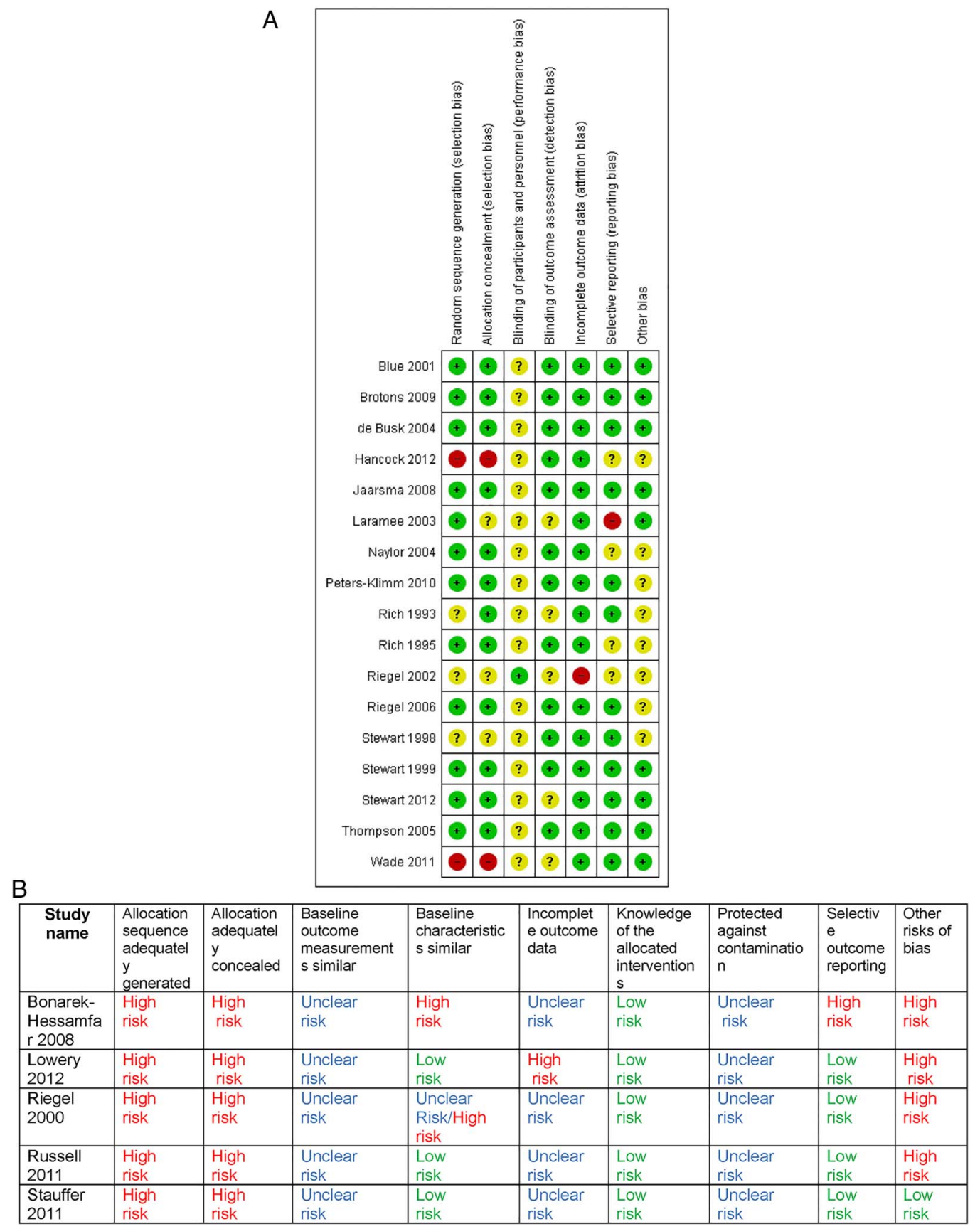

Figure 2 (A) Risk of bias of included randomised controlled trials. (B) Effective Practice and Organisation of Care (EPOC) risk of bias for non-randomised controlled trials.

longer time-related effect was difficult to assess due to lack of data (figure 4B-D).

The one study comparing $\mathrm{CM}$ with specialist clinic care reported that $\mathrm{CM}$ patients accumulated 592 and clinic patients 547 all-cause hospitalisations $(\mathrm{p}=0.087)$ associated with 3067 vs 4410 days of hospital stay ( $p<0.01$ for rate and duration of hospital stay). ${ }^{32}$

\section{Community-initiated CM}

Of the four community-initiated trials comparing CM with usual care, two did not report $\operatorname{LOS}^{38}{ }^{43}$ one reported median values in favour of $\mathrm{CM}^{44}$ and the remaining one reported a mean reduction in $\operatorname{LOS}^{45}$ (table 2). The one comparative trial between community-initiated $\mathrm{CM}$, and telehealth and $\mathrm{CM}$ did not report any useful data. ${ }^{42}$

\section{Intervention components}

Fourteen intervention components were identified and grouped as per the CM definition in methods and prevalence determined for hospital-initiated and community-initiated $\mathrm{CM}$ studies with a usual care control group (see tables 1 and 2 and online supplementary appendix 2$){ }^{7}$ 
A Overall for hospital -initiated $\mathrm{CM}$

CM Usual Care Rate Ratio Rate Ratio

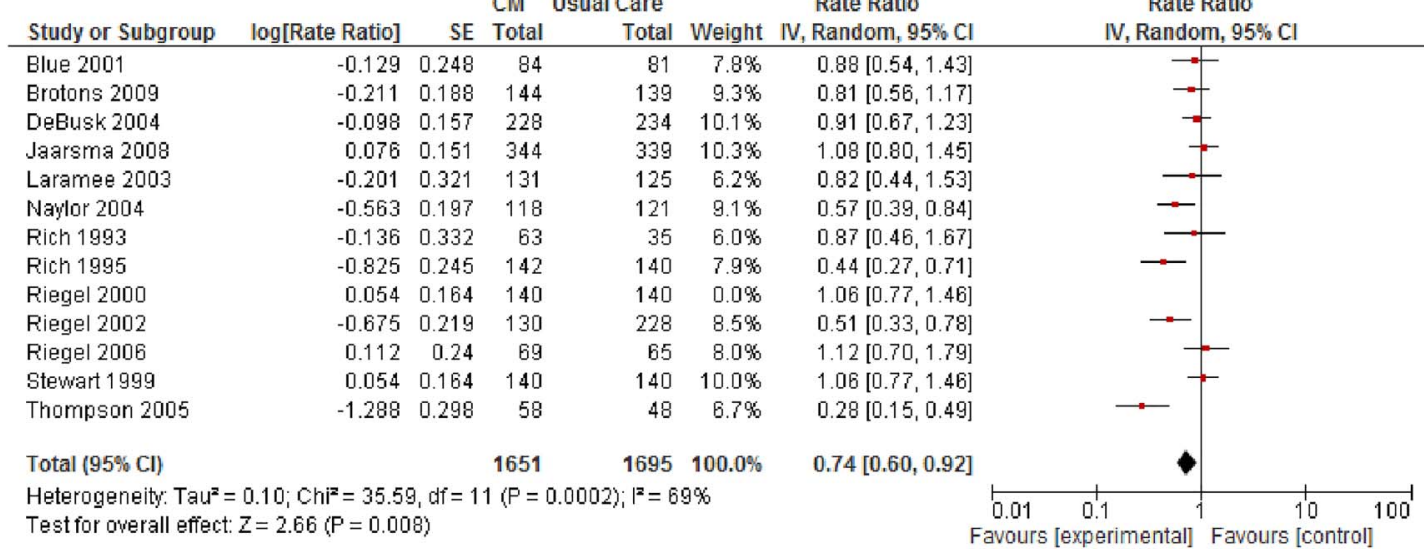

Sensitivity analysis: (Riegel 2002 removed) $I^{2}=68 \%$ RR $0.77[0.61,0.96] p=0.02$

B 3 month data for hospital -initiated CM

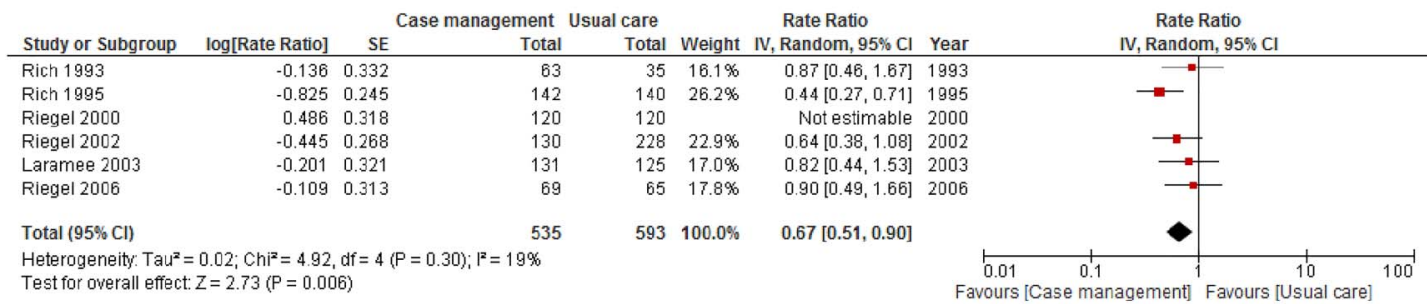

Sensitivity analysis: (Riegel 2002 removed) $I^{2}=39 \%$ RR $0.70[0.48,1.01] \mathrm{p}=0.06$

\section{6 month data for hospital -initiated CM}

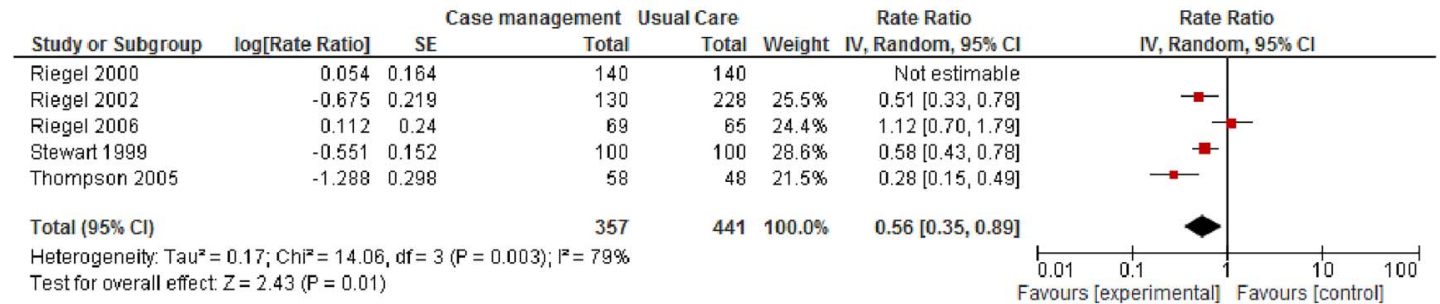

Sensitivity analysis: (Riegel 2002 removed) $\mathrm{I}^{2}=85 \% \mathrm{RR} 0.57[0.30,1.21] \mathrm{p}=0.1$

D $12-18$ months for hospital -initiated CM

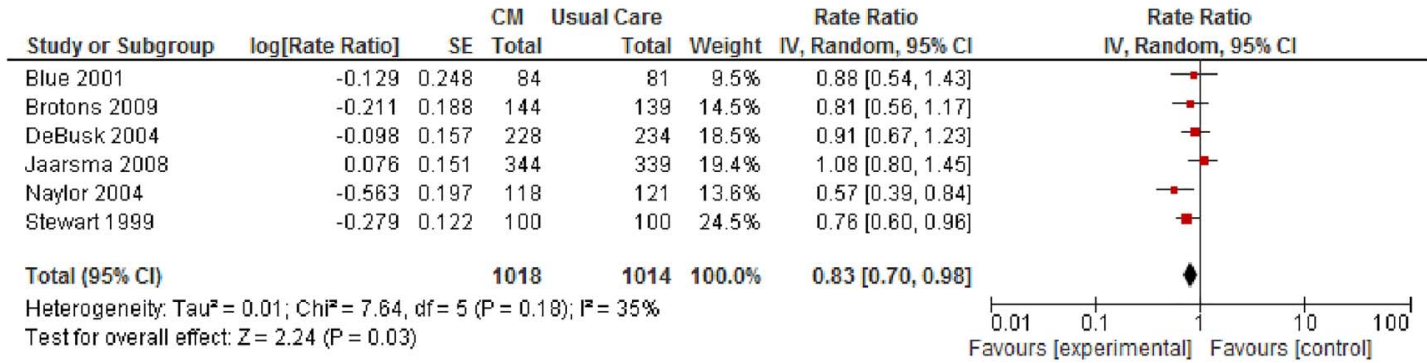

Figure 3 Chronic heart failure (CHF) admissions data. CM, case management. 
A Overall for hospital-initiated CM

\begin{tabular}{|c|c|c|c|c|c|c|c|c|c|c|c|}
\hline \multirow[b]{2}{*}{ Study or Subgroup } & \multicolumn{3}{|c|}{ Case management } & \multicolumn{3}{|c|}{ Usual care } & \multirow[b]{2}{*}{ Weight } & \multirow{2}{*}{$\begin{array}{l}\text { Mean Difference } \\
\text { IV, Random, } 95 \% \mathrm{Cl}\end{array}$} & \multirow{2}{*}{\multicolumn{3}{|c|}{$\begin{array}{l}\text { Mean Difference } \\
\text { IV, Random, } 95 \% \mathrm{Cl}\end{array}$}} \\
\hline & Mean & SD & Total & Mean & SD & Total & & & & & \\
\hline Blue 2001 & 3.42 & 12.2 & 82 & 7.46 & 16.6 & 75 & $2.5 \%$ & $-4.04[-8.63,0.55]$ & & & \\
\hline Laramee 2003 & 6.9 & 6.5 & 131 & 9.5 & 9.8 & 125 & $9.1 \%$ & $-2.60[-4.65,-0.55]$ & $=-$ & & \\
\hline Rich 1993 & 4.3 & 1.1 & 63 & 5.7 & 2 & 35 & $21.2 \%$ & $-1.40[-2.12,-0.68]$ & $=$ & & \\
\hline Rich 1995 & 3.9 & 10 & 142 & 6.2 & 11.4 & 140 & $6.8 \%$ & $-2.30[-4.80,0.20]$ & - & & \\
\hline Riegel 2000 & 1.31 & 3.77 & 120 & 1.08 & 3.46 & 120 & $0.0 \%$ & $0.23[-0.69,1.15]$ & & & \\
\hline Riegel 2002 & 1.1 & 3.1 & 130 & 2.1 & 4.6 & 228 & $20.3 \%$ & $-1.00[-1.80,-0.20]$ & 름 & & \\
\hline Riegel 2006 & 3.65 & 7.8 & 69 & 3.4 & 7.1 & 65 & $6.8 \%$ & $0.25[-2.27,2.77]$ & $\rightarrow$ & & \\
\hline Stewart 1999 & 0.9 & 0.9 & 100 & 2.9 & 2.9 & 100 & $22.5 \%$ & $-2.00[-2.60,-1.40]$ & $\stackrel{-}{-1}$ & & \\
\hline Stewart 2012 & 4.96 & 8.57 & 143 & 3.62 & 6.36 & 137 & $10.9 \%$ & $1.34[-0.42,3.10]$ & & & \\
\hline Total $(95 \% \mathrm{Cl})$ & & & 860 & & & 905 & $100.0 \%$ & $-1.28[-2.04,-0.52]$ & $\bullet$ & & \\
\hline \multicolumn{9}{|c|}{$\begin{array}{l}\text { Heterogeneity: } \text { Tau }^{2}=0.58 ; \mathrm{Chi}^{2}=18.75, \mathrm{df}=7(\mathrm{P}=0.009) ; \mathrm{I}^{2}=63 \% \\
\text { Test for overall effect: } Z=3.29(\mathrm{P}=0.001)\end{array}$} & -10 & & 10 \\
\hline
\end{tabular}

Sensitivity analysis: (Riegel 2002 removed) $I^{2}=14 \%$ MD $-1.76[-2.29,-1.23] p<0.00001$

B 3 month data for hospital -initiated CM

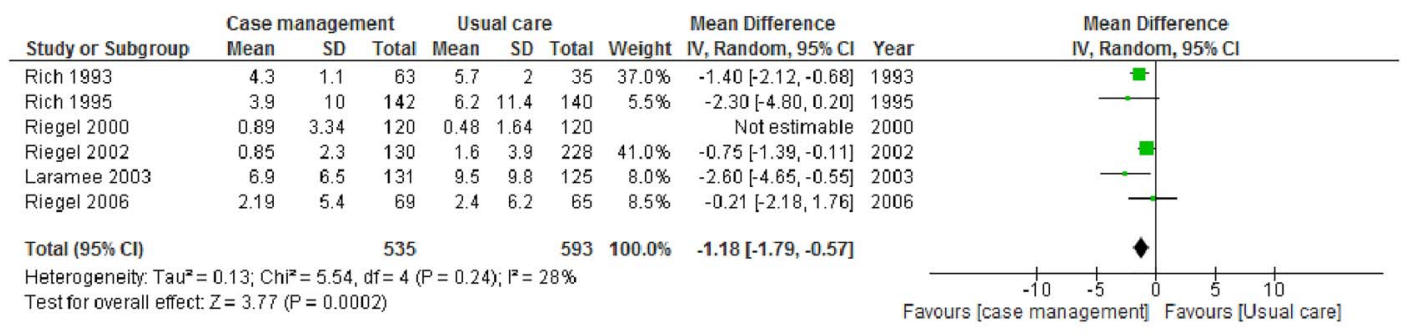

Sensitivity analysis: (Riegel 2002 removed) $I^{2}=6 \%$ MD $-1.46[-2.15,-0.77] p<0.0001$

C 6 month data for hospital-initiated CM

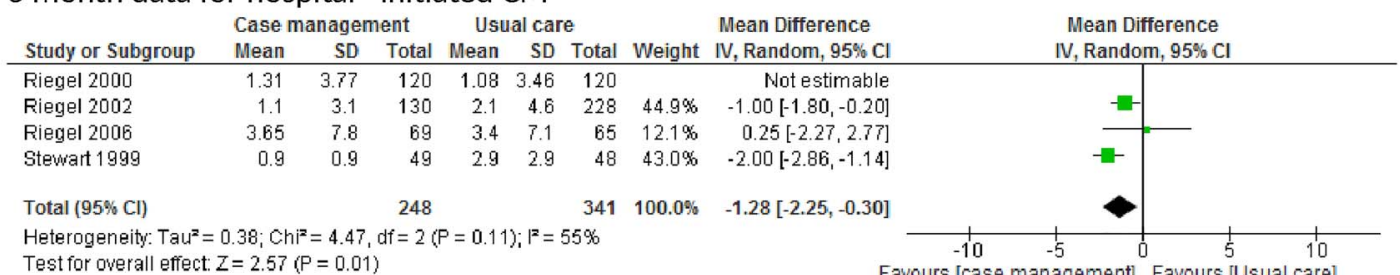

Sensitivity analysis: (Riegel 2002 removed so $n=2$ ) $I^{2}=63 \% M D-1.20[-3.31,0.91] p=0.26$

Figure 4 Chronic heart failure (CHF) length of hospital stay. CM, case management.

\section{Hospital-initiated CM}

Data from individual studies which contained components of family involvement showed an overall reduction in hospital readmissions in comparison with usual care and a reduction in hospital readmissions observationally in comparison with interventions which did not contain these components (rate ratio of 0.56 (0.34 to 0.92); $\mathrm{p}=0.003$ ). However, post hoc analysis comparing these studies, in which the component was present with those studies in which the component was absent, did not yield any statistically significant differences $(\mathrm{p}=0.15$; see online supplementary appendix $2 \mathrm{a}$ ). The same calculations for medication review; referral to other services; and assessment of home environment, CM meetings and patientdirected access did not indicate any specific effect of these components of hospital-initiated $\mathrm{CM}$ on rates of admission (see online supplementary appendix 2b-g)
The majority of the interventions included education/ self-management, and there were insufficient data from studies without this component to allow comparison.

\section{Community-initiated CM}

There were insufficient data to conduct any subgroup analysis on any of the remaining components of hospitalinitiated CM, community-initiated studies or the LOS data.

\section{Outpatient healthcare resources}

Only six of the included studies measured outpatient resource use. In some studies, outpatient resource data were all-cause and not HF-specific. In some studies, primary and secondary use was combined. ${ }^{23} 2435384245$ Two of these studies also reported ED attendance. ${ }^{23} 42$ All but one of these studies reported no difference between intervention and control group for these measures with the 
Table 3 Available cost data from studies $(n=9)$

\begin{tabular}{|c|c|}
\hline Study & Cost data intervention vs control (NS=not statistically significant) \\
\hline \multirow[t]{3}{*}{ Rich et $a l^{15}$} & 3-month data \\
\hline & Study intervention cost US\$216 per person \\
\hline & Hospital readmissions $\$ 2178$ vs $\$ 3236 p=0.03$ \\
\hline \multirow[t]{6}{*}{ Stewart et $a l^{17}$} & 6-month data \\
\hline & Cost of study intervention $\$ A \$ 190$ per person \\
\hline & Mean cost of hospital-based care \\
\hline & $\$ 3200$ (1800-4600), \$5400 (3200-6800) NS \\
\hline & Cost of community-based care \\
\hline & $\$ 620$ (460 740), \$680 (550 800) NS \\
\hline \multirow[t]{5}{*}{ Stewart et al ${ }^{19}$} & 6 -month and 18-month data \\
\hline & 6 months \\
\hline & Total hospital-based care $\$ A \$ 490300$ vs $\$ A 922600$ NS \\
\hline & 18-month data \\
\hline & Total hospital-based care $\$ 5100$ (6800) vs $\$ 10600$ (13 000) NS \\
\hline \multirow[t]{3}{*}{ Laramee et $a l^{22}$} & 3-month data \\
\hline & Total care costs \\
\hline & Mean(US\$) 23054 vs25 536 NS \\
\hline \multirow[t]{12}{*}{ Naylor et al ${ }^{24}$} & 12-month data \\
\hline & CHF readmissions US $\$ 175840$ vs US $\$ 498110 ?$ \\
\hline & Physician's office (outpatients) $\$ 4549, \$ 5169$ NS \\
\hline & ER visits $\$ 1780$ vs $\$ 5650 \mathrm{NS}$ \\
\hline & Home visits (all cause) \\
\hline & Visiting nurse $\$ 11021, \$ 64531 p<0.001$ \\
\hline & APN $\$ 104019$ vs 0 \\
\hline & Physical therapist $\$ 7120$ vs $\$ 10918$ NS \\
\hline & Social worker $\$ 178$ vs $\$ 534$ NS \\
\hline & Home health aides $\$ 9167$ vs $\$ 11081$ NS \\
\hline & Total home visits $\$ 138649$ vs $\$ 97.883 p<0.001$ \\
\hline & Total costs $\$ 725903, \$ 1163810$ NS \\
\hline \multirow[t]{5}{*}{ Stewart et al ${ }^{32}$} & 12-18-month data \\
\hline & Costs per patient \\
\hline & $\$ A \$ 1813(220)$ vs $A \$ 1829(174)$ NS \\
\hline & Total costs \\
\hline & $\$ \mathrm{~A} \$ 3.93$ million vs $\mathrm{A} \$ 5.53$ million $\mathrm{p}=0.03$ for median costs per day \\
\hline \multirow[t]{4}{*}{ Riegel et $a l^{35}$} & 3-month and 6-month data \\
\hline & Total costs \\
\hline & 3 months US $\$ 632$ (2378) vs US $\$ 317$ (1188) NS \\
\hline & 6 months $\$ 1024$ (3017) vs $\$ 686$ (2225) NS \\
\hline \multirow[t]{5}{*}{ Riegel et $a l^{36}$} & 1-month, 3-month and 6-month data \\
\hline & HF inpatient costs all NS \\
\hline & 1 month US\$1012 (4022) vs US\$2830 (13 896) \\
\hline & 3-month $\$ 3045$ (7784) vs $\$ 4130$ (14 468) \\
\hline & 6-month $\$ 5567$ (13 137) vs $\$ 6151$ (16 650) \\
\hline \multirow[t]{2}{*}{ Stauffer et $a l^{37}$} & 1-month data \\
\hline & $\begin{array}{l}\text { 'Under the current payment system, the intervention reduced the hospital financial contribution on } \\
\text { average by US } \$ 227 \text { for each Medicare patient with HF' }\end{array}$ \\
\hline
\end{tabular}

exception of Lowery et $a l^{45}$ which showed a statistically significant greater use of outpatient resources in the usual care group (optional primary care visits 1 year 16.75 (13.62); 10.43 (9.6), $\mathrm{p}<0.001$; 2 year 14.27 (11.98); 9.35 (9.97), $\mathrm{p}<0.001)$.

\section{Costs}

Nine of the 17 hospital-initiated trials described cost data (table 3). Of these, six reported no statistically significant difference between CM (3-month or 6-month duration) and usual care,, 171822242635 and three reported costs in favour of $\mathrm{CM}$ although data from Stauffer et $a \hat{l}^{7}$ was brief. ${ }^{152}$ One of these was $12-18$ months ${ }^{32}$ and two were 3 months in duration. It was difficult from the intervention descriptions to determine their intensity. There were no cost data reported from the community-initiated trials.

\section{DISCUSSION}

This systematic review confirms that hospital-initiated CM can be successful in reducing unplanned hospital 
readmissions, and reducing LOS in hospital in the short term for people with HF. There were only five communityinitiated CM studies (three RCTs and two NRCTs) of which four were at high risk of bias. This limited evidence suggests no effect of community-initiated CM on hospital admissions. A minority of trials report cost comparisons with usual care and most of those show no difference. There were limited data on the effect of CM on other healthcare resources.

Many factors are likely to modify the effect of CM on use of emergency care seen in these studies. It is generally accepted that $\mathrm{CM}$ is more appropriate for people with severe HF and poorer general health. However, it was difficult to compare the health status of the study participants in hospital-initiated and community-initiated trials as in some studies there was little detail, others gave median and mean figures for NYHA status, and the presentation format and detail of comorbidities varied. All the included studies have been conducted within the past 12 years, so it is important to put these results in the context of overall improved treatment and reduction in hospital admissions since the early 1990s. ${ }^{4-6}$

Seventeen studies described hospital-initiated CM and five described community-initiated $\mathrm{CM}$ of $\mathrm{HF}$, although often the participants were identified via hospital clinic records. Overall, the meta-analysis showed that CM reduced readmissions and hospital LOS. This may be explained by the fact that in most of the trials the participants were identified via hospital contact, and therefore were likely to have had a recent exacerbation of their $\mathrm{HF}$ and to be at increased risk of readmission in the postdischarge period. In addition, it is likely that interventions are acting at a time of highest risk as reflected by $\mathrm{HF}$ mortality in first year of diagnosis. ${ }^{4}$ Therefore, once they were assessed and given extra support, they were stable for a period of time. Previous work by Roland $e t a l^{46}$ suggests that admission rates in people aged 65 with two or more emergency admissions in 12 months fall in subsequent years without any intervention and account for fewer than $10 \%$ of admissions in the following year, and thus effectiveness of admission avoidance schemes cannot be judged by tracking admission rates without careful comparison with a control group. The data from trials of community-initiated CM was lacking both in the number of studies, and the fact there were limited useable data that showed no effect on unplanned hospital admissions. It is likely that these patients were likely to be in more stable health. ${ }^{41}$

A metareview of a wide range of HF disease management programmes by Savard et $a l^{47}$ reports that nine previous systematic reviews (2001-2009) identified significant reductions in $\mathrm{HF}$ admissions with reductions in risk ranging from $30 \%$ to $56 \%$. However, the authors caution that these reviews are limited by inadequate reporting in the population, setting, intervention and comparator components. They report that reviewers have not taken into account statistical, clinical and methodological heterogeneity in interventions. ${ }^{47}$ Our review focused specifically on CM avoiding some of these limitations and indicates a reduction in $\mathrm{HF}$ readmissions with hospital-initiated CM in the range of $10-30 \%$.

Wakefield $e t a l^{48}$ in 2013 looked at common components of a range of $\mathrm{HF}$ care programmes focusing mainly on disease management and education investigated in RCTs, and 10/35 of the discussed studies were included in our review. They described patient education, symptom management by health professionals and by patients, and medication adherence strategies as the most commonly occurring elements of care. A literature review by Jaarsma et $a l^{49}$ looked at 70 'home care' controlled studies (mostly RCTs) which encompassed 9 of our included CM studies covering a wide range of approaches such as telemedicine, hospital at home and health buddies for patients with HF. They identified a multidisciplinary team, continuity of care, care plans, optimising titration of medication, education/counselling of patients and caregivers and increased access as important. Unfortunately, we had insufficient data to perform subanalysis on the component of education/ self-management.

Previous systematic reviews have investigated the role of the lay caregiver in $\mathrm{HF}$ patient management. ${ }^{50-52}$ These suggest that better relationship quality and communication were related to reduced mortality, increased health status and less distress, and improved patient selfcare outcomes. Our review adds to this evidence base by suggesting that more family involvement in CM may also reduce unscheduled readmissions.

Education about HF and about its pharmacological and non-pharmaceutical treatment has been well reviewed both as an individual approach and as part of complex interventions, and is considered to be essential for improving many patient outcomes. ${ }^{49} 5354$ A recent mixed-method study suggests asking patients with HF to write down their learning needs before the education increases their chances of receiving education based on their individual needs. ${ }^{55}$ Qualitative interviews with health professionals caring for patients with HF suggest that communication with, and education by specialist nurses facilitated by continuity of care is essential to good care of patients with HF. The authors also highlight the role of the specialist nurse in multidisciplinary team communication and functioning, essentially describing the role of the specialist nurse as a case manager. ${ }^{56}$

Our review of CM suggests that the evidence for its cost-effectiveness is lacking with most studies that have performed cost comparisons with usual care show no advantage. Previous work by de Bruin et $a l^{57}$ looked at cost-effectiveness of disease management for a range of chronic conditions and concluded that the data are most positive for $\mathrm{HF}$ with five out of the eight included studies showing cost-effectiveness.

\section{Strengths and limitations}

The contribution of our high-quality systematic review to the above is that we have focused on CM which is based 
on nurse coordinated multicomponent care of patients which is applicable to the primary care-based health systems such as that in the UK. We have focused on HF (re)admissions and LOS as opposed to all-cause data which many of the previous reviews have used.

By examining the components of CM, we have a profile of the components most likely to lead to the success of CM of patients with $\mathrm{HF}$ in terms of reducing (re)admissions and hospital LOS. Our review has highlighted the potential importance of family involvement albeit in post hoc analysis.

The limitations of this review are that majority of the community-initiated CM studies were of low quality with the exception of one low risk of bias RCT, and provided limited evidence. While funnel plot analysis was not appropriate with our data, we acknowledge that there may be publication bias on this topic. ${ }^{58}$ This was counteracted by the fact that the hospital-initiated studies comprised of predominantly community-based CM. There is a lack of cost data and analysis in the included papers. This point needs to be emphasised for future trials. It is possible that cost-effectiveness will be more likely with intervention for patients with more severe $\mathrm{HF}$.

\section{CONCLUSIONS}

Hospital-initiated CM reduces unplanned hospital admissions, and LOS for people with HF in the short term. Cost data are limited. There was limited evidence for community-initiated CM which suggested it does not reduce hospital admission. Further research is needed to determine the individual components of $\mathrm{CM}$ that contribute to reduced admissions.

Contributors ALH is the main systematic reviewer, and worked across all stages of the review from inception to completed draft. RJ is the cardiology and primary care expertise, and worked on screening, selection of studies, commenting on analysis, and development and checking of final document content. AK is the second reviewer, involved in screening, selection, data checking and commenting on developing and final document content. RWM is the statistical expertise, advising on data analysis and commenting on the developing and final document content. SP is the primary care and admission avoidance expertise, and advised throughout project, third reviewer for screening process and commenting on the developing and final document content.

Funding This project was funded by the National School of Primary Health Care project no.238.

Competing interests None declared.

Provenance and peer review Not commissioned; externally peer reviewed.

Data sharing statement Full data extraction tables and data analysis files are available on request.

Open Access This is an Open Access article distributed in accordance with the Creative Commons Attribution Non Commercial (CC BY-NC 4.0) license, which permits others to distribute, remix, adapt, build upon this work noncommercially, and license their derivative works on different terms, provided the original work is properly cited and the use is non-commercial. See: http:// creativecommons.org/licenses/by-nc/4.0/

\section{REFERENCES}

1. Cardiovascular disease statistics 2014 British Heart Foundation. https://www.bhf.org.uk/ /media/files/publications/research/bhf cvd-statistics-2014_web_2.pdf on the 7/10/2015.

2. Quality and Outcomes Framework Achievement, prevalence and exceptions data 2012/13. 29 October 2013. http://www.hscic.gov. uk/catalogue/PUB12262/qual-outc-fram-12-13-rep.pdf on the 7/10/2015.

3. de Giuli F, Khaw KT, Cowie MR, et al. Incidence and outcome of persons with a clinical diagnosis of heart failure in a general practice population of 696,884 in the United Kingdom. Eur J Heart Fail 2005;7:295-302.

4. Stewart S, Maclntyre K, Hole DJ, et al. More 'malignant' than cancer? Five-year survival following a first admission for heart failure. Eur J Heart Fail 2001;3:315-22.

5. Stewart S, Ekman I, Ekman T, et al. Population impact of heart failure and the most common forms of cancer: a study of 1162309 hospital cases in Sweden (1988 to 2004). Circ Cardiovasc Qual Outcomes 2010;3:573-80.

6. Jhund PS, Macintyre K, Simpson CR, et al. Long-term trends in first hospitalization for heart failure and subsequent survival between 1986 and 2003: a population study of 5.1 million people. Circulation 2009;119:515-23.

7. Ross S, Curry N, Goodwin N. Case management. What is it and how it can best be implemented. London, UK: The Kings Fund, 2011. http://www.kingsfund.org.uk/publications/case_management. $\mathrm{html}$ on the7/10/15.

8. Department of Health. Supporting people with long term conditions liberating the talents of nurses who care for people with long term conditions. London, UK: Department of Health, 2005. http://www.dh. gov.uk/en/Publicationsandstatistics/Publications/ PublicationsPolicyAndGuidance/DH_4102469 on 01/12/2011.

9. Huntley AL, Thomas R, Mann M, et al. Is case management effective in reducing the risk of unplanned hospital admissions for older people? A systematic review and meta-analysis. Fam Pract 2013;30:266-75.

10. Purdy S. Interventions to reduce unplanned hospital admission: a series of systematic reviews. http://www.bristol.ac.uk/media-library/ sites/primaryhealthcare/migrated/documents/unplannedadmissions. pdf on 07/10/2015.

11. List of OECD Member countries. http://www.oecd.org/about/ membersandpartners/list-oecd-member-countries.htm on 07/10/ 2015.

12. Chapter 8: Assessing risk of bias in included studies http:// handbook.cochrane.org/chapter_8/8_assessing_risk_of_bias_in_ included_studies.htm on 07/10/2015.

13. Chapter 9-9.7 Sensitivity analyses. http://handbook.cochrane.org/ chapter_9/9_7_sensitivity_analyses.htmon07/10/2015.

14. Rich MW, Vinson JM, Sperry JC, et al. Prevention of readmission in elderly patients with congestive heart failure: results of a prospective, randomized pilot study. $J$ Gen Intern Med 1993:8:585-90.

15. Rich MW, Beckham V, Wittenberg C, et al. A multidisciplinary intervention to prevent the readmission of elderly patients with congestive heart failure. N Engl J Med 1995;333: 1190-5.

16. Rich MW, Gray DB, Beckham V, et al. Effect of a multidisciplinary intervention on medication compliance in elderly patients with congestive heart failure. Am J Med 1996;101:270-6.

17. Stewart S, Pearson S, Luke CG, et al. Effects of home-based intervention on unplanned readmissions and out-of-hospital deaths. J Am Geriatr Soc 1998;46:174-80.

18. Stewart S, Marley JE, Horowitz JD. Effects of a multidisciplinary, home-based intervention on unplanned readmissions and survival among patients with chronic congestive heart failure: a randomised controlled study. Lancet 1999;354:1077-83.

19. Stewart S, Horowitz JD. Home-based intervention in congestive heart failure: long-term implications on readmission and survival. Circulation 2002;105:2861-6.

20. Blue L, Lang E, McMurray JJ, et al. Randomised controlled trial of specialist nurse intervention in heart failure. BMJ 2001;323:715-18.

21. Riegel B, Carlson B, Kopp Z, et al. Effect of a standardized nurse case-management telephone intervention on resource use in patients with chronic heart failure. Arch Intern Med 2002;162:705-12.

22. Laramee AS, Levinsky SK, Sargent J, et al. Case management in a heterogeneous congestive heart failure population: a randomized controlled trial. Arch Intern Med 2003;163:809-17.

23. DeBusk RF, Miller NH, Parker KM, et al. Care management for low-risk patients with heart failure: a randomized, controlled trial. Ann Intern Med 2004;141:606-13. 
24. Naylor MD, Brooten DA, Campbell RL, et al. Transitional care of older adults hospitalized with heart failure: a randomized, controlled trial. J Am Geriatr Soc 2004;52:675-84.

25. McCauley KM, Bixby MB, Naylor MD. Advanced practice nurse strategies to improve outcomes and reduce cost in elders with heart failure. Dis Manag 2006;9:302-10.

26. Riegel B, Carlson B, Glaser D, et al. Randomized controlled trial of telephone case management in Hispanics of Mexican origin with heart failure. J Card Fail 2006;12:211-19.

27. Thompson DR, Roebuck A, Stewart S. Effects of a nurse-led, clinic and home-based intervention on recurrent hospital use in chronic heart failure. Eur J Heart Fail 2005;7:377-84.

28. Jaarsma T, van der Wal MH, Lesman-Leegte I, et al. Coordinating Study Evaluating Outcomes of Advising and Counseling in Heart Failure $(\mathrm{COACH})$ Investigators. Effect of moderate or intensive disease management program on outcome in patients with heart failure: Coordinating Study Evaluating Outcomes of Advising and Counseling in Heart Failure (COACH). Arch Intern Med 2008;168:316-24

29. Postmus D, Pari AA, Jaarsma T, et al. A trial-based economic evaluation of 2 nurse-led disease management programs in heart failure. Am Heart J 2011;162:1096-104.

30. Inglis SC, Pearson S, Treen S, et al. Extending the horizon in chronic heart failure: effects of multidisciplinary, home-based intervention relative to usual care. Circulation 2006;114:2466-73.

31. Brotons C, Falces C, Alegre J, et al. Randomized clinical trial of the effectiveness of a home based intervention in patients with heart failure: the IC-DOM study. Rev Esp Cardiol 2009;62:400-8.

32. Stewart S, Carrington MJ, Marwick TH, et al. Impact of home versus clinic-based management of chronic heart failure: the WHICH? (Which Heart Failure Intervention Is Most Cost-Effective \& Consumer Friendly in Reducing Hospital Care) multicenter, randomized trial. J Am Coll Cardiol 2012;60:1239-48.

33. Stewart S, Carrington MJ, Horowitz JD, et al. Prolonged impact of home versus clinic based management of chronic heart failure: extended follow-up of a pragmatic, multicentre randomized trial cohort. Int J Cardiol 2014;174:600-10.

34. Stewart S, Carrington MJ, Chan YK. Prolonged benefits of nurse-led, home-based intervention versus a specialist heart failure clinic: extended follow-up of the WHICH? Trial Cohort. Eur J Heart Failure 2014;16.

35. Riegel B, Carlson B, Glaser D, et al. Which patients with heart failure respond best to multidisciplinary disease management? J Card Fail 2000;6:290-9.

36. Russell D, Rosati RJ, Sobolewski S, et al. Implementing a transitional care program for high-risk heart failure patients: findings from a community-based partnership between a certified home healthcare agency and regional hospital. J Healthc Qual 2011;33:17-23.

37. Stauffer BD, Fullerton C, Fleming N, et al. Effectiveness and cost of a transitional care program for heart failure: a prospective study with concurrent controls. Arch Intern Med 2011;171:1238-43.

38. Peters-Klimm F, Campbell S, Hermann K, et al. Case management for patients with chronic systolic heart failure in primary care: the HICMan exploratory randomised controlled trial. Trials 2010;11:56

39. Peters-Klimm F, Müller-Tasch T, Schellberg D, et al. Rationale, design and conduct of a randomised controlled trial evaluating a primary care-based complex intervention to improve the quality of life of heart failure patients: HICMan (Heidelberg Integrated Case Management). BMC Cardiovasc Disord 2007;7:25.

40. Freund $\mathrm{F}$, Baldauf $\mathrm{A}$, Muth $\mathrm{C}$, et al. [Practice-based home visit and telephone monitoring of chronic heart failure patients: rationale, design and practical application of monitoring lists in the HICMan trial]. Z Evid Fortbild Qual Gesundhwes 2011;105:434-45.
41. Peters-Klimm F, Campbell S, Müller-Tasch T, et al. Primary care-based multifaceted, interdisciplinary medical educational intervention for patients with systolic heart failure: lessons learned from a cluster randomised controlled trial. Trials 2009;10:68.

42. Wade MJ, Desai AS, Spettell CM, et al. Telemonitoring with case management for seniors with heart failure. Am J Manag Care 2011;17:e71-9.

43. Hancock HC, Close H, Mason JM, et al. Feasibility of evidencebased diagnosis and management of heart failure in older people in care: a pilot randomised controlled trial. BMC Geriatr 2012;12:70.

44. Bonarek-Hessamfar M, Benchimol D, Lauribe $\mathrm{P}$, et al. Multidisciplinary network in heart failure management in a community based population: results and benefits at 2 years. Int $J$ Cardiol 2009;134:120-2.

45. Lowery J, Hopp F, Subramanian U, et al. Evaluation of a nurse practitioner disease management model for chronic heart failure: a multi-site implementation study. Congest Heart Fail 2012;18:64-71.

46. Roland M, Dusheiko M, Gravelle $\mathrm{H}$, et al. Follow up of people aged 65 and over with a history of emergency admissions: analysis of routine admission data. BMJ 2005;330:289-92.

47. Savard LA, Thompson DR, Clark AM. A metareview of evidence on heart failure disease management programs: the challenges of describing and synthesizing evidence on complex interventions. Trials 2011;12:194.

48. Wakefield BJ, Boren SA, Groves PS, et al. Heart failure care management programs: a review of study interventions and meta-analysis of outcomes. J Cardiovasc Nurs 2013;28:8-19.

49. Jaarsma T, Brons M, Kraai I, et al. Components of heart failure management in home care; a literature review. Eur J Cardiovasc Nurs 2013;12:230-41

50. Hooker SA, Grigsby ME, Riegel B, et al. The impact of relationship quality on health-related outcomes in heart failure patients and informal family caregivers: an integrative review. $J$ Cardiovasc Nurs 2015;30(Suppl 1):S52-63.

51. Buck HG, Harkness K, Wion R, et al. Caregivers' contributions to heart failure self-care: a systematic review. Eur J Cardiovasc Nurs 2015;14:79-89.

52. Clark AM, Spaling M, Harkness K, et al. Determinants of effective heart failure self-care: a systematic review of patients' and caregivers' perceptions. Heart 2014;100:716-21.

53. Spatola CF, Cocchieri A, De Marinis MG, et al. Educational interventions in patients with heart failure: a review of the literature. Ig Sanita Pubbl 2013;69:557-74.

54. Boyde M, Turner C, Thompson DR, et al. Educational interventions for patients with heart failure: a systematic review of randomized controlled trials. J Cardiovasc Nurs 2011;26:E27-35.

55. Ross A, Ohlsson U, Blomberg $\mathrm{K}$, et al. Evaluation of an intervention to individualise patient education at a nurse-led heart failure clinic: a mixed-method study. J Clin Nurs 2015;24:1594-602.

56. Glogowska M, Simmonds R, McLachlan S, et al. Managing patients with heart failure: a qualitative study of multidisciplinary teams with specialist heart failure nurses. Ann Fam Med 2015;13:466-71.

57. de Bruin SR, Heijink R, Lemmens LC, et al. Impact of disease management programs on healthcare expenditures for patients with diabetes, depression, heart failure or chronic obstructive pulmonary disease: a systematic review of the literature. Health Policy 2011;101:105-21.

58. Chapter 10 Recommendations on testing for funnel plot asymmetry. http://handbook.cochrane.org/chapter_10/10_4_3_1 recommendations on testing for funnel plot asymmetry.htm on 07/10/2015. 